\title{
Article \\ Fatigue Damage Map of AZ31B-F Magnesium Alloys under Multiaxial Loading Conditions
}

\author{
Vitor Anes $1,2, * \mathbb{C}$, Luis Reis ${ }^{2, *}$ and Manuel Freitas ${ }^{2}$ \\ 1 Instituto Superior de Engenharia de Lisboa, Instituto Politécnico de Lisboa, 1959-007 Lisboa, Portugal \\ 2 IDMEC, Instituto Superior Técnico, Universidade de Lisboa, 1049-001 Lisboa, Portugal; \\ manuel.freitas@tecnico.ulisboa.pt \\ * Correspondence: vitor.anes@isel.pt (V.A.); luis.g.reis@tecnico.ulisboa.pt (L.R.); Tel.: +351-916-222-536 (V.A.)
}

check for updates

Citation: Anes, V.; Reis, L.; Freitas,

M. Fatigue Damage Map of AZ31B-F Magnesium Alloys under Multiaxial Loading Conditions. Metals 2021, 11, 1616. https://doi.org/10.3390/ met11101616

Academic Editors: Hamid Jahed and Andrew Gryguć

Received: 18 September 2021

Accepted: 8 October 2021

Published: 11 October 2021

Publisher's Note: MDPI stays neutral with regard to jurisdictional claims in published maps and institutional affiliations.

Copyright: (c) 2021 by the authors. Licensee MDPI, Basel, Switzerland. This article is an open access article distributed under the terms and conditions of the Creative Commons Attribution (CC BY) license (https:// creativecommons.org/licenses/by/ $4.0 /)$.

\begin{abstract}
In this work, the mechanical behavior of the AZ31B-F magnesium alloy under cyclic loading is analyzed with the goal of contributing to the advancement of its use in the design of AZ31B-F components and structures. To achieve this goal, an experimental program was implemented to evaluate the cyclic response of the AZ31B-F under specific proportional loads with different stress amplitude ratios. Afterwards, regression methods were applied to extend the experimental data to a wide range of proportional loads. As a result, the AZ31B-F damage map, a material property that stablishes the damage scale between normal and shear stresses for finite life loading regimes, was obtained. In addition, a safety factor was developed for the AZ31B-F material when subjected to proportional loading. The achieved results have a direct application in mechanical design of components/structures made of AZ31B-F contributing to its reliability.
\end{abstract}

Keywords: AZ31B-F magnesium alloy; multiaxial fatigue; fatigue damage; experimental testing

\section{Introduction}

Nowadays, sustainability is a major concern for society. Over the years, industry in general has evolved without really thinking about the environmental impact of its strategies. However, societies are beginning to realize that change is needed, especially in the transportation industry, which today has a strong impact on the sustainability of planet Earth by contributing to the increase in greenhouse gas emissions [1-3]. In this sense, alternative strategies have been developed to reduce gas emissions by reducing the weight of transportation structures. In this context, the replacement of steels and aluminum alloys with magnesium alloys has been strongly considered. Magnesium alloys are the lightest structural metals. They are 33\% lighter than aluminum alloys and $75 \%$ lighter than steels.

In fact, the use of magnesium alloys in the transportation industry is not new. The first magnesium alloys developed were used in the automotive and aircraft industries, especially for castings, but the low corrosion resistance of these alloys dampened the expectations placed in them [4]. In the meantime, new structural magnesium alloys with better corrosion resistance and mechanical strength have been developed [5-7]. These new properties have encouraged the use of magnesium alloys for applications other than castings. Magnesium alloys have a very different mechanical behavior than steels or even aluminum alloys. The hexagonal close-packed structure gives these alloys various properties such as polarity, twinning, mechanical behavior dependent on loading conditions, anisotropy due to slip-twin interactions, and different stress-strain behavior in tension and compression, making the mechanical behavior of these alloys quite different from that of other structural metals [8-12].

In this sense, it is extremely important to develop tools that characterize this mechanical behavior in order to be used in mechanical design. This is because the tools normally used in the design of steel or aluminum alloy components and structures do not take into account the mechanical behavior of magnesium alloys, especially in fatigue. 
Many papers have been published in the literature on the fatigue strength of magnesium alloys under uniaxial loading conditions [13-16], and new methods have been proposed to evaluate the fatigue strength of magnesium alloys under such loading conditions. For example, Liu et al. [17] proposed a new method for evaluating AZ31B using thermal indicators, which employs an infrared thermography technique to analyze the temperature variations under cyclic loading, which in turn is used to estimate fatigue strength. This result is of particular interest in very high cycle fatigue testing where the high selfheating temperatures present additional challenges due to the high testing frequencies [18]. Despite these advances, there is very little work for multiaxial loading conditions [19-22]. This is of particular concern as the loads in practice are usually multiaxial, i.e., the components and structures are usually subjected to normal and shear stresses with different amplitudes over time and different loading sequences. These loading conditions are very different from those simulated in the laboratory when a uniaxial SN curve is evaluated. Nevertheless, uniaxial loading testing remains the preferred approach for characterizing the fatigue strength of magnesium alloys. In fact, the uniaxial loading case is important in that one needs a reference, called the SN curve, to estimate fatigue life. However, the problem is that the link between fatigue strength under multiaxial loading and uniaxial fatigue strength is missing. The evaluation of this relationship in magnesium alloys is a complex and interesting challenge, strongly influenced by the cyclic behavior of closed microstructures typically found in magnesium alloys.

One way to make this connection is to develop some sort of equivalence between multiaxial and uniaxial loading conditions. This allows multiaxial stress conditions to be reduced to a single equivalent stress, which is used in conjunction with the uniaxial SN curve to estimate the fatigue strength of a given material under multiaxial loading [21-23]. This equivalence between loads is always done by calculating shear and normal stresses to obtain an equivalent normal stress or an equivalent shear stress. This calculation must always take into account the extent of damage between these stresses in order to calculate this equivalence.

In fact, this calculation is necessary because normal and shear stresses have different damage scales, for both static and cyclic loading conditions [21,23]. For illustration, known information can be used, e.g., the fatigue limit of the uniaxial shear curve $\mathrm{SN}$ is always lower than the fatigue limit of the uniaxial normal curve $\mathrm{SN}$ for a given material. This means that the fatigue strength varies according to the type of stress, in this sense, the damage scale of shear stresses is always different from the damage scale of normal stresses. Moreover, the von Mises formula for equivalent stresses in static or quasi-static loading defines $\sqrt{ } 3$ as the scaling factor between normal and shear stresses. From this, it can be seen that normal and shear stresses have different damage scales and that a constant scaling factor is used when calculating an equivalent stress to account for both types of stresses. The problem is that this scaling factor is not constant for materials subjected to cyclic loading and varies depending on the type of material, stress level, and multiaxial loading conditions, such as the ratio of shear to normal stress. This scaling factor is much more complex than what has been considered in the conventional multiaxial fatigue models, which consider this scaling factor as a constant. It can be assumed that this scaling factor is a material property that can be measured by experimental tests [21]. In this sense, this work aims to evaluate the scale of damage between cyclic loads (normal and shear) for magnesium alloy AZ31B-F under proportional loading conditions. This evaluation will be based on experimental tests and the result will be presented as a damage map, which can be used as a material property representing the AZ31B-F damage scale between shear and normal stresses for multiaxial loading conditions, which is a novelty in the mechanical characterization of magnesium alloys. Moreover, the damage map of AZ31B-F obtained in this work is compared with the damage map of AISI 4140, a high strength steel studied in previous works [21,23]. This comparison allows conclusions to be drawn about the influence of the microstructure on the damage scale between shear and normal stresses. 


\section{Materials and Methods}

This paper investigates the fatigue strength behavior of AZ31B-F under multiaxial fatigue testing conditions. The objective is to evaluate the damage map of the AZ31B-F. This is a function that relates the damage scale of normal stresses with respect to shear stresses or vice versa. For this purpose, an experimental program has been implemented to obtain the necessary data to calculate the damage map. Then the experimental results are computed to derive the damage scale between shear and normal stresses, and then a fitting is made to model the experimental tests. As a result, a function with two variables is obtained, which provides the damage scale for the stress paths considered in the experimental program and for the stress paths not considered. The determined damage map can be used in the fatigue design of AZ31B-F magnesium alloy components and structures subjected to multiaxial loads.

\subsection{Material}

The chemical composition of magnesium alloy AZ31B-F is mainly composed of $97 \%$ magnesium (Mg), 3\% aluminum ( $\mathrm{Al}$ ) and 1\% zinc ( $\mathrm{Z}$ ). The letter $\mathrm{B}$ indicates that this alloy was the second to be developed, and $\mathrm{F}$ is a code designation meaning "As fabricated". Table 1 shows the complete chemical composition of AZ31B-F.

Table 1. Typical AZ31B chemical composition.

\begin{tabular}{cccccccccc}
\hline Element & $\mathbf{A l}$ & $\mathbf{Z n}$ & $\mathbf{M n}$ & $\mathbf{F e}$ & $\mathbf{N i}$ & $\mathbf{C u}$ & $\mathbf{C a}$ & $\mathbf{S i}$ & $\mathbf{M g}$ \\
\hline Weight $(\%)$ & 3.1 & 1.05 & 0.54 & 0.0035 & 0.0007 & 0.0008 & 0.04 & 0.1 & Balance \\
\hline
\end{tabular}

In this composition, aluminum aims to increase the strength of the alloy, manganese produces relatively harmless compounds and improves corrosion resistance by controlling the solubility of iron, which is a very harmful impurity because it reduces corrosion resistance, and zinc, like aluminum, aims to improve the mechanical strength of the alloy. AZ31B-F is available in various forms such as plates, sheets, and bars. It is an alternative to aluminum alloys as it has a high strength to weight ratio and is widely available compared to other magnesium alloys. This alloy is easy to machine but is flammable and requires the use of a lubricant during machining to lower the temperature at the point of machining. It can also be formed by preheating to $260^{\circ} \mathrm{C}$ and can be welded using arc welding techniques. The main applications are aerospace and general commercial. AZ31B-F is a wrought alloy with typical tensile strength, yield strength in tension and compressive strength values of $290 \mathrm{MPa}$, $203 \mathrm{MPa}$, and $97 \mathrm{MPa}$, respectively. Table 2 summarizes the typical mechanical properties for these alloys.

Table 2. AZ31B-F mechanical properties, data from [17].

\begin{tabular}{cc}
\hline Microstructure Type & HC \\
\hline Poisson's ratio & 0.35 \\
Density $\left(\mathrm{Kg} / \mathrm{m}^{3}\right)$ & 1770 \\
Hardness (HV) & 86 \\
Tensile strength (MPa) & 290 \\
Yield strength (MPa) & 203 \\
Elongation (\%) & 14 \\
Young's modulus (GPa) & 45 \\
$\sigma_{\mathrm{f}}^{\prime}-$ Fatigue strength coefficient (MPa) & 450 \\
b-Fatigue strength coefficient & -0.12 \\
$\varepsilon_{\mathrm{f}}^{\prime}-$ Fatigue ductility coefficient & 0.26 \\
c-Fatigue ductility exponent & -0.71 \\
\hline
\end{tabular}

\subsection{Experimental Program}

Fatigue tests were carried out through a biaxial servo-hydraulic fatigue testing machine, Instron 8874 (Instron, Norwood, MA, USA) 25 kN/100 Nm, under stress control 
according to the ASTM E466 Standard. Figure 1 shows the geometry of the specimens and the corresponding dimensions. The specimens were fabricated with a $\mathrm{CNC}$ machine from extruded AZ31BF rods, with their longitudinal direction coinciding with the extrusion direction. Due to the hexagonal, compact microstructure of AZ31BF and due to the extrusion process, some anisotropy in the radial direction maybe present in the fabricated samples. The samples were polished with sandpaper of decreasing grit size until they were mirror polished.

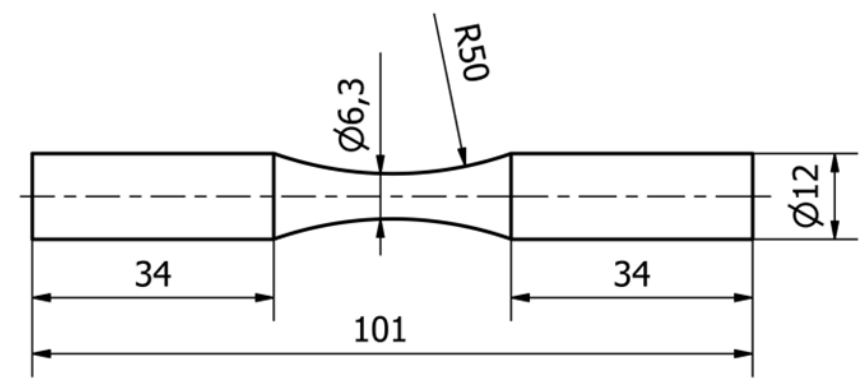

Figure 1. Specimen geometry and respective dimensions in [mm].

The experimental program carried out includes a series of proportional multiaxial fatigue tests with different ratios of normal to shear amplitude and $r=-1$ in both loading channels, i.e., no mean stresses were considered in these loads. The tests were conducted at room temperature and a loading frequency of $4 \mathrm{~Hz}$, which corresponds to the upper limit established for the strain rate of magnesium alloys in fatigue tests. The criterion for completion of the tests was complete separation of the specimen. Figure 2 shows the stress paths plotted in the von Mises stress space.

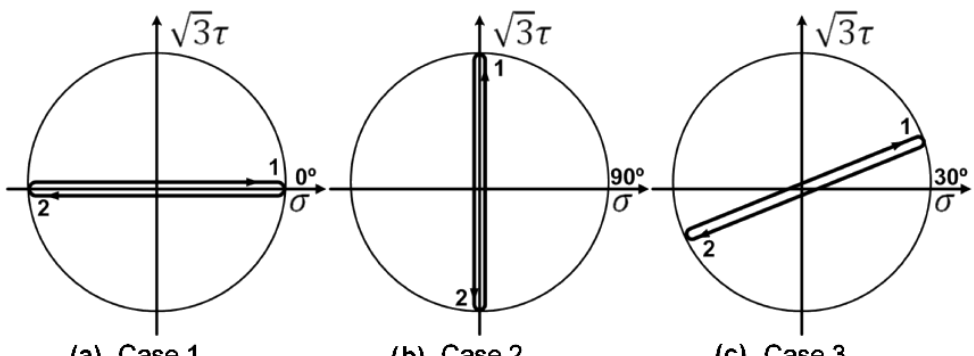

(a) Case 1

(b) Case 2

(c) Case 3

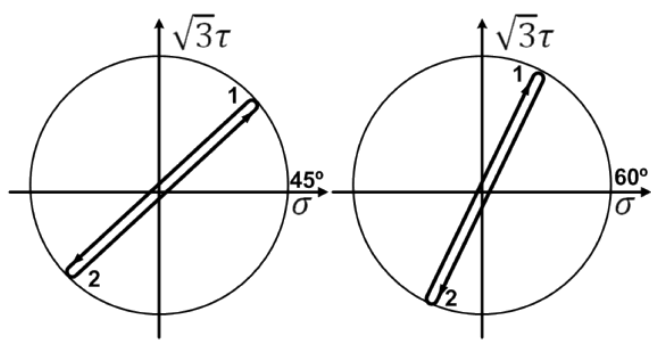

(d) Case 4

(e) Case 5

Figure 2. Proportional loadings performed in the fatigue life experimental program, (a) Case 1 and (b) Case 2 are the uniaxial normal and shear loading cases, (c) Case 3 is the proportional loading with a shear to normal stress ratio equal to $\tan \left(30^{\circ}\right)$, (d) Case 4 is the proportional loading with a shear to normal stress ratio equal to $\tan \left(45^{\circ}\right)$, and (e) Case 5 is the proportional loading with a shear to normal stress ratio equal to $\tan \left(60^{\circ}\right)$.

Figure 2a,b represent the two uniaxial load paths, normal and shear (PT and PS), and Figure 2c-e represent three proportional loads (PP30, PP45, and PP60) with different ratios of normal to shear amplitude. PP30 means that the arctangent of the ratio of shear to normal amplitude gives a $30^{\circ}$ angle, PP45 gives a $45^{\circ}$ angle, and PP60 gives a $60^{\circ}$ angle. 
Increasing the angle means increasing the amplitude of the shear stress and decreasing the amplitude of the normal stress. In this way, it becomes possible to distinguish proportional loads according to the predominance of shear stresses over normal stresses and vice versa on a given proportional load path. Figure 3 summarizes the amplitudes of the normal and shear stresses with $\mathrm{R}=-1$ in used in the experiments for each loading path.

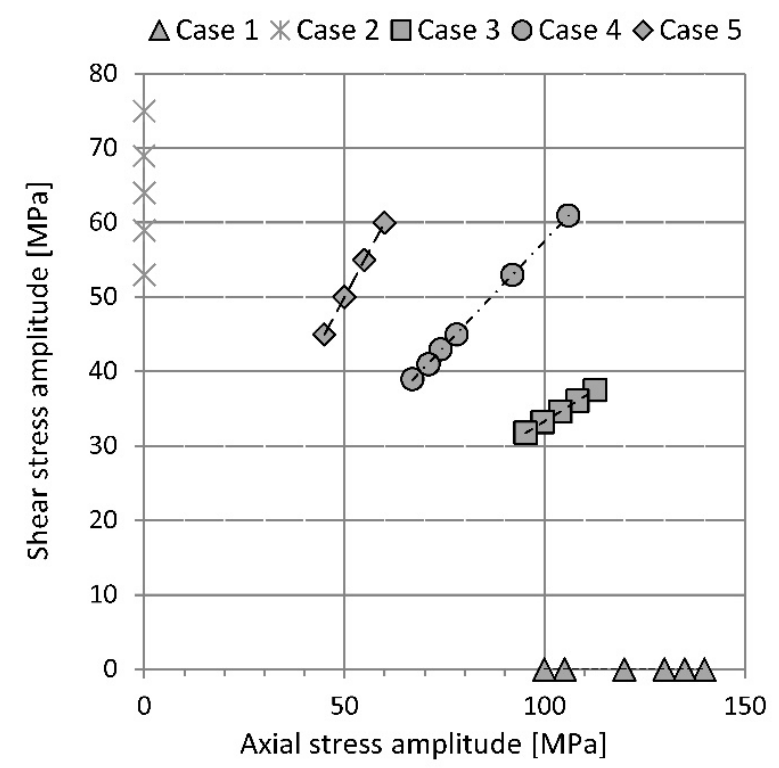

Figure 3. Normal and shear stress amplitudes with $\mathrm{R}=-1$ used in experiments.

\subsection{Fatigue Damage Map Assessment Method}

In this work, the damage map for the magnesium alloy AZ31B-F was constructed following the procedures published by Anes et al. [21] for the high strength steel 42CrMo4. The main idea behind this procedure is based on the concept that the normal and shear components of a proportional load have different damage scales, and both can be calculated together to obtain an equivalent shear stress with a fatigue damage scale equivalent to that found in the uniaxial shear stress amplitudes. Figure 4 illustrates this idea by comparing two SN curves of a given material. One is the uniaxial shear curve SN and the other is the $\mathrm{SN}$ curve of a given proportional load represented by the amplitudes of normal and shear stress. The comparison between these two SN curves is made by considering the stress amplitudes at both loads that lead to the same fatigue damage, i.e., that lead to the same number of load cycles at failure. In this way, it can be assumed that these stress amplitudes are equivalent as they lead to the fracture of the material at the same number of load cycles, i.e., they lead to the same fatigue life result.

To illustrate this in Figure 4, fatigue damage was considered at $10^{5}$ loading cycles where the uniaxial shear amplitude $\mathrm{SN}$ is between the proportional normal and shear stress amplitudes, which is always the case for any material subjected to proportional loading. As can be seen, the proportional shear stress amplitude represented by the segment $A B$ is not sufficient to cause fatigue failure at $10^{5}$ cycles of loading. For this purpose, it is necessary to add an additional shear stress amplitude equal to the segment $B C$ to achieve the uniaxial shear stress amplitude leading to the rupture at $10^{5}$ load cycles, represented by the segment $\mathrm{AC}$ in Figure 4. In this sense, the damage of the additional shear stress amplitude BC required to cause the fatigue rupture at $10^{5}$ load cycles is caused by the normal stress amplitude represented by the segment AD. However, this segment is much larger than the BC segment, which means that there is a different damage scale between normal and shear stress amplitudes, because the damage caused by the AD (nor-mal) segment is equal to the damage caused by the BC (shear) segment, but these segments have different lengths, so their scales must be different. 


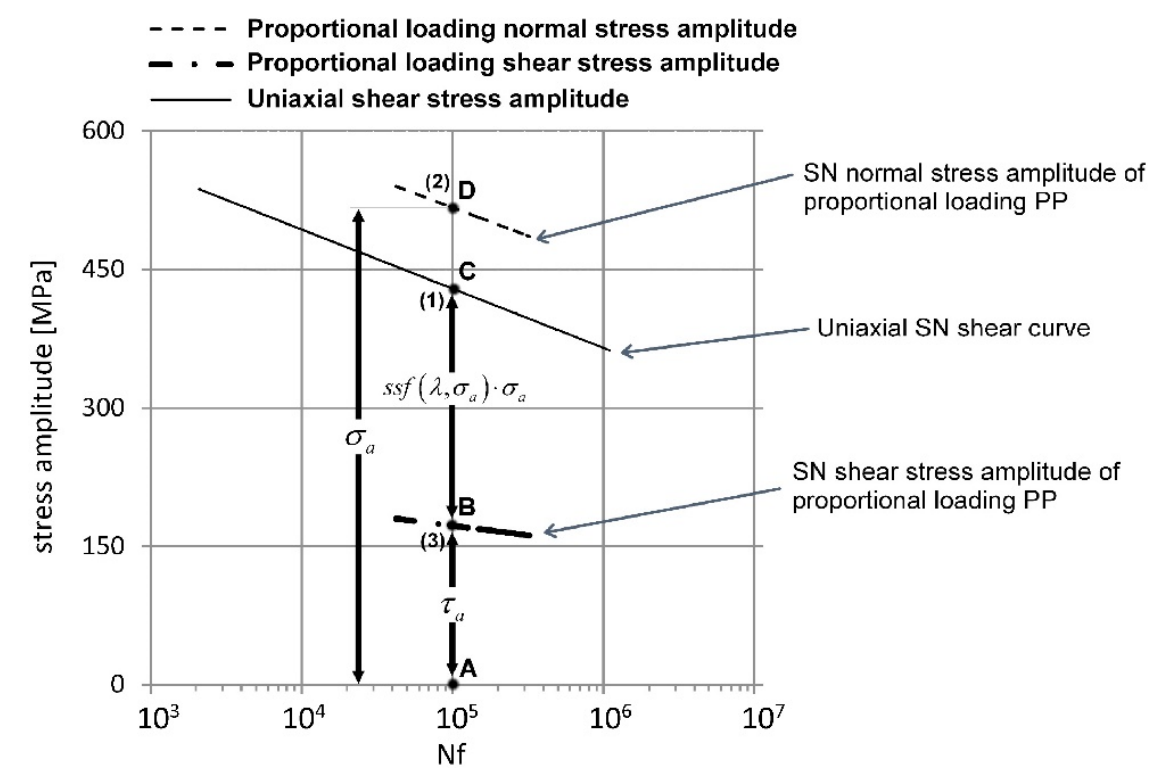

Figure 4. Different damage scales representation for normal and shear stress amplitudes considering a given fatigue strength $(\mathrm{Nf})$.

To convert the normal damage scale into a shear damage scale, Anes et al. [21] use the stress scale factor (ssf) given by the ratio of BC segment to AC segment. From experiments, the authors found that this factor varies as a function of the normal stress amplitude $\left(\sigma_{a}\right)$ and the ratio of shear stress $\left(\tau_{a}\right)$ to normal stress amplitude, represented by $\lambda$ in Figure 4 , where $\lambda=\tau_{a} / \sigma_{a}$. With this in mind, the authors established the stress paths shown in Figure 2 to investigate the variation of the stress scale factor in $42 \mathrm{CrMo} 4$ for different proportional stress paths and different normal stress amplitudes. In order to estimate the ssf factor for proportional stress paths that were not considered in the experiments, the authors performed a fitting of the experimental results obtained in this way to the function $\operatorname{ssf}\left(\lambda, \sigma_{a}\right)$, which is the fatigue damage map that updates the fatigue damage scale of normal stresses to the damage scale of shear stresses. With this damage map, it becomes possible to calculate the ssf equivalent shear stress, Equation (1), and then estimate the fatigue life using the uniaxial shear curve $\mathrm{SN}$.

$$
\tau_{\text {eqv }}=\tau_{a}+s s f\left(\lambda, \sigma_{a}\right) \cdot \sigma_{a}
$$

The purpose of this paper is to determine the $\operatorname{ssf}\left(\lambda, \sigma_{a}\right)$ function (fatigue damage map) for the magnesium alloy AZ31B-F and to compare the results with those obtained by Anes et al. [21] for 42CrMo4.

\section{Results and Discussion}

\subsection{S-N Experimental Results}

Table 3 shows the experimental results obtained for each loading path shown in Figure 2 and the stress amplitudes shown in Figure 3. In run-out situations, i.e., cases where the specimen did not fracture as a result of the applied loading, the number of loading cycles $\mathrm{Nf}$ is presented as $10^{6}$ cycles. 
Table 3. Az31B-F experimental fatigue data for proportional loading paths, Cases 1 to 5.

\begin{tabular}{cccc}
\hline Loading Case & Normal Stress (MPa) & Shear Stress (MPa) & Nf \\
\hline & 140 & & 13,164 \\
Case 1 & 135 & & 22,873 \\
PT & 130 & & 38,102 \\
& 120 & & 62,352 \\
& 105 & & 721,573 \\
& 100 & 75 & $1,000,000$ \\
\hline Case 2 & & 69 & 88,871 \\
PS & & 64 & 128,769 \\
& & 59 & 227,808 \\
& & 53 & 388,236 \\
Case 3 & & 37.53 & $1,000,000$ \\
PP30 & 112.58 & 36.08 & 65,318 \\
& 108.25 & 34.64 & 84,432 \\
& 103.92 & 33.2 & 170,311 \\
& 99.59 & 31.75 & 366,799 \\
& 95.26 & 61 & $1,000,000$ \\
\hline Case 4 & 106 & 53 & 16,800 \\
PP45 & 92 & 45 & 46,874 \\
& 78 & 43 & 138,986 \\
PP60 & 74 & 41 & 242,685 \\
& 71 & 39 & 353,718 \\
& 67 & 60 & $1,000,000$ \\
\hline & 60 & 55 & 52,110 \\
& 55 & 50 & 94,116 \\
& 50 & 45 & $1,000,000$ \\
\hline
\end{tabular}

Similar to the procedure developed by Anes et al. [21] for the analysis and calculation of fatigue data, a trend line approach over the experimental results was used to correlate the amplitudes of normal and shear stress. To perform this correlation, the trend line equations for each stress component (normal and shear stress) were obtained (see Figure 5). The trend lines were represented by dashed lines in the graphs and the respective equation was shown near these lines.

Figure $5 b-d$ show the results of the fatigue data for loading cases 3,4 , and 5 , respectively. In each case, the biaxial loading is represented by two trend lines, one representing the axial stress component and the other the shear stress component, as described in Section 2.3. In addition, the pure shear results (loading case 2) are also presented together with each proportional loading case and case 1 . The aim is to use Case 2 as a reference, as shown in Figure 4. From the results shown in Figure 5, it can be concluded that the shear stress amplitude of each biaxial loading compared to the reference line $\mathrm{SN}$ (Case 2) is clearly insufficient to cause fatigue failure, i.e., the trend lines of shear stress amplitudes of loading cases 3 to 5 are always lower than the reference case. This means that the part of the damage that is absent in the shear loading is caused by the axial component. Moreover, the axial trend lines in cases 3 and 4 are above the SN line of case 2. This result clearly indicates that normal and shear loading have different damage magnitudes. If only this loading component was used in the reference trend line equation to estimate the fatigue life, the result would be shorter than the experimental results. The opposite is observed in loading case 5 , the axial trend line of the biaxial loading is lower than the reference case. Table 4 summarizes the trend line equations obtained based on the experimental data for each loading path. The trend lines have a power law format that typically fits well with the fatigue behavior, with acceptable $\mathrm{R}^{2}$ values between 0.95 and 0.98 . 


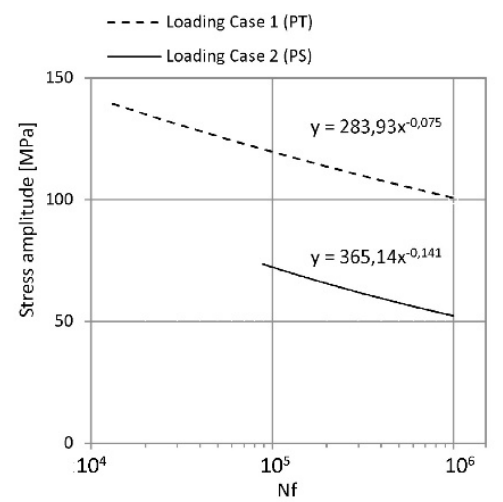

(a)

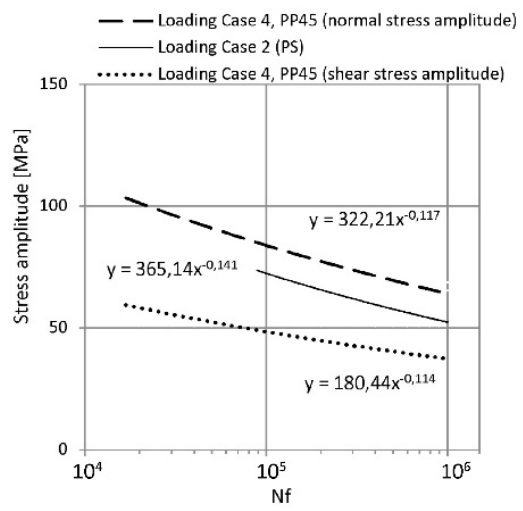

(c)

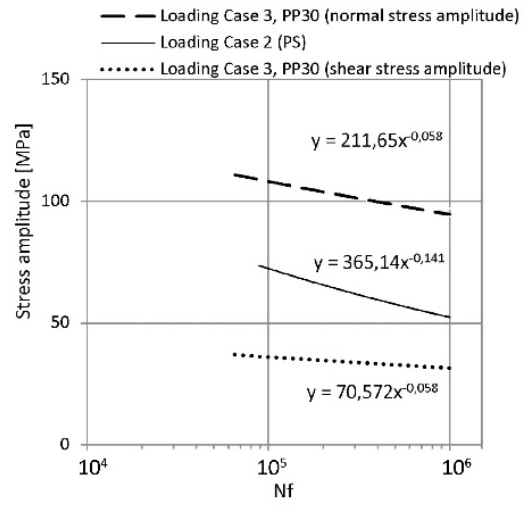

(b)

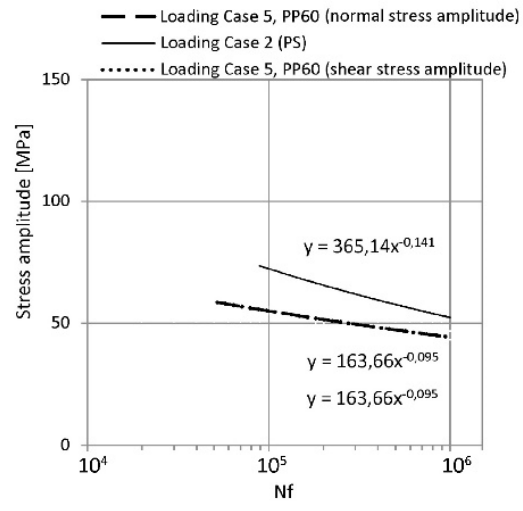

(d)

Figure 5. S-N experimental data and respective trend lines representation for loading Cases 1 to 5. (a) Case 1 PT and Case 2 PS, (b) Case 3 PP30, (c) Case 4 PP45, and (d) Case 5 PP60.

Table 4. S-N trend lines for normal and shear loading components of loading cases 1 to 5 experimentally evaluated for AZ31B-F.

\begin{tabular}{ccc}
\hline Case & $\lambda=\tau_{\mathrm{a}} / \sigma_{\mathrm{a}}$ & Trend Line [MPa] \\
\hline \multirow{2}{*}{1} & 0 & $\sigma_{\mathrm{a}}=283.93\left(\mathrm{~N}_{\mathrm{f}}\right)^{-0.075}$ \\
& $\tau_{\mathrm{a}}=0$ \\
2 & $\infty$ & $\sigma_{\mathrm{a}}=0$ \\
& \multirow{2}{*}{0.33} & $\tau_{\mathrm{a}}=365.14\left(\mathrm{~N}_{\mathrm{f}}\right)^{-0.141}$ \\
3 & 0.33 & $\sigma_{\mathrm{a}}=211.65\left(\mathrm{~N}_{\mathrm{f}}\right)^{-0.058}$ \\
& $\tau_{\mathrm{a}}=70.572\left(\mathrm{~N}_{\mathrm{f}}\right)^{-0.058}$ \\
4 & 0.56 & $\sigma_{\mathrm{a}}=322.22\left(\mathrm{~N}_{\mathrm{f}}\right)^{-0.117}$ \\
& 1 & $\tau_{\mathrm{a}}=180.44\left(\mathrm{~N}_{\mathrm{f}}\right)^{-0.114}$ \\
5 & 1 & $\sigma_{\mathrm{a}}=163.66\left(\mathrm{~N}_{\mathrm{f}}\right)^{-0.095}$ \\
& $\tau_{\mathrm{a}}=163.66\left(\mathrm{~N}_{\mathrm{f}}\right)^{-0.095}$ \\
\hline
\end{tabular}

\subsection{Stress Scale Factor (ssf) Determination Based on Experimental Results}

In this section, the stress scaling factor is calculated as described in Section 2.3 using the trend lines summarized in Table 4 . The calculated results for loading cases 1 and 3 to 5 are shown in Tables $5-8$. In these tables, the first row shows the trend line equations used to calculate the stress amplitudes as a function of the number of cycles to failure (column 1), and the last position of the first row shows the reasoning used to calculate the ssf parameter using the numbers in the columns containing the stress amplitudes calculated using the respective trend lines. For example, if we consider Table 5, Case 3, to calculate the ssf parameter for $\mathrm{Nf}=10^{5}$ cycles, the uniaxial shear stress amplitude is first evaluated using the experimental trend line $\tau_{\mathrm{a}}=365.14\left(10^{5}\right)^{\wedge}(-0.141)$, which yields 
$72 \mathrm{MPa}$, as shown in the second column (1). Afterwards, the normal stress amplitude of the PP30 loading path is also evaluated for $\mathrm{Nf}=10^{5}$ cycles using the corresponding trend line, $\sigma_{\mathrm{a}}=211.65\left(10^{5}\right)^{\wedge}(-0.058)$, which yields $109 \mathrm{MPa}$, shown in the third column (2). Next, the shear stress amplitude of the PP30 loading path for $\mathrm{Nf}=10^{5}$ cycles is evaluated using the trend line, $\tau_{\mathrm{a}}=70.572\left(10^{5}\right)^{\wedge}(-0.058)$, which yields $36 \mathrm{MPa}$, shown in the fourth column (3). To evaluate the ssf parameter at $\mathrm{Nf}=10^{5}$ cycles, the expression shown in the last column is used, i.e., (72-36) $/ 109$ that gives the value of 0.33 . The data presented in Tables $5-8$ have been compiled in Table 9 to find a model that better describes these data using regression methods.

Table 5. ssf results for Case 1-AZ31B-F.

\begin{tabular}{cccc}
\hline Nf & $\begin{array}{c}(\mathbf{1}) \\
\text { Pure Shear (Case 2) }\end{array}$ & $\begin{array}{c}(\mathbf{2}) \\
\text { Pure tension (Case 1) }\end{array}$ & $\begin{array}{c}\mathbf{s s f}=(\mathbf{1}) /(\mathbf{2}) \\
\boldsymbol{\sigma}_{\mathbf{a}}=\mathbf{3 6 5 . 1 4}(\mathbf{N f})^{\wedge}(-\mathbf{0 . 1 4 1})[\mathbf{M P a}]\end{array}$ \\
\hline $10^{3}$ & 138 & 169 & $138 / 169=0.82$ \\
$10^{4}$ & 100 & 142 & 0.70 \\
$5 \times 10^{4}$ & 79 & 126 & 0.63 \\
$10^{5}$ & 72 & 120 & 0.60 \\
$5 \times 10^{5}$ & 57 & 106 & 0.54 \\
$10^{6}$ & 52 & 101 & 0.52 \\
\hline
\end{tabular}

Table 6. ssf results for Case 3-AZ31B-F.

\begin{tabular}{|c|c|c|c|c|}
\hline $\mathrm{Nf}$ & $\begin{array}{c}(1) \\
\text { Pure Shear (Case 2) } \\
\tau_{\mathrm{a}}=365.14(\mathrm{Nf}) \wedge(-0.141) \\
{[\mathrm{MPa}]}\end{array}$ & $\begin{array}{c}(2) \\
\text { Normal (Case 3) } \\
\sigma_{\mathrm{a}}=211,65(\mathrm{Nf})^{\wedge}(-0.058) \\
{[\mathrm{MPa}]}\end{array}$ & $\begin{array}{c}(3) \\
\text { Shear }(\text { Case 3) } \\
\tau_{\mathrm{a}}=70,572(\mathrm{Nf})^{\wedge}(-0.058) \\
{[\mathrm{MPa}]}\end{array}$ & $\begin{array}{c}\text { ssf }= \\
((1)-(3)) /(2)\end{array}$ \\
\hline $10^{3}$ & 138 & 142 & 47 & 0.64 \\
\hline $10^{4}$ & 100 & 124 & 41 & 0.47 \\
\hline $5 \times 10^{4}$ & 79 & 113 & 38 & 0.37 \\
\hline $10^{5}$ & 72 & 109 & 36 & 0.33 \\
\hline $5 \times 10^{5}$ & 57 & 99 & 33 & 0.25 \\
\hline $10^{6}$ & 52 & 95 & 32 & 0.21 \\
\hline
\end{tabular}

Table 7. ssf results for Case 4-AZ31B-F.

\begin{tabular}{|c|c|c|c|c|}
\hline Nf & $\begin{array}{c}(1) \\
\text { Pure Shear (Case 2) } \\
\tau_{\mathrm{a}}=365.14(\mathrm{Nf})^{\wedge}(-0.141) \\
{[\mathrm{MPa}]}\end{array}$ & $\begin{array}{c}(2) \\
\text { Normal (Case 4) } \\
\sigma_{\mathrm{a}}=322,21(\mathrm{Nf})^{\wedge}(-0.117) \\
{[\mathrm{MPa}]}\end{array}$ & $\begin{array}{c}(3) \\
\text { Shear }(\text { Case } 4) \\
\tau_{\mathrm{a}}=180,44(\mathrm{Nf})^{\wedge}(-0.114) \\
{[\mathrm{MPa}]}\end{array}$ & $\begin{array}{c}\text { ssf }= \\
((1)-(3)) /(2)\end{array}$ \\
\hline $10^{3}$ & 138 & 144 & 82 & 0.39 \\
\hline $10^{4}$ & 100 & 110 & 63 & 0.33 \\
\hline $5 \times 10^{4}$ & 79 & 91 & 53 & 0.30 \\
\hline $10^{5}$ & 72 & 84 & 49 & 0.28 \\
\hline $5 \times 10^{5}$ & 57 & 69 & 40 & 0.24 \\
\hline $10^{6}$ & 52 & 64 & 37 & 0.23 \\
\hline
\end{tabular}

Table 8. ssf results for Case 5-AZ31B-F.

\begin{tabular}{|c|c|c|c|c|}
\hline Nf & $\begin{array}{c}(1) \\
\text { Pure Shear (Case 2) } \\
\tau_{\mathrm{a}}=365.14(\mathrm{Nf}) \wedge(-0.141) \\
{[\mathrm{MPa}]}\end{array}$ & $\begin{array}{c}(2) \\
\text { Normal (Case 5) } \\
\sigma_{\mathrm{a}}=163,66(\mathrm{Nf})^{\wedge}(-0.095) \\
{[\mathrm{MPa}]}\end{array}$ & $\begin{array}{c}(3) \\
\text { Shear (Case 5) } \\
\tau_{\mathrm{a}}=163,66(\mathrm{Nf}) \wedge(-0.095) \\
{[\mathrm{MPa}]}\end{array}$ & $\begin{array}{c}\text { ssf }= \\
((1)-(3)) /(2)\end{array}$ \\
\hline $10^{3}$ & 138 & 85 & 85 & 0.62 \\
\hline $10^{4}$ & 100 & 68 & 68 & 0.46 \\
\hline $5 \times 10^{4}$ & 79 & 59 & 59 & 0.36 \\
\hline $10^{5}$ & 72 & 55 & 55 & 0.31 \\
\hline $5 \times 10^{5}$ & 57 & 47 & 47 & 0.22 \\
\hline $10^{6}$ & 52 & 44 & 44 & 0.18 \\
\hline
\end{tabular}


Table 9. ssf results for all loading cases-AZ31B-F.

\begin{tabular}{ccc}
\hline$\sigma_{\mathbf{a}}$ & $\lambda=\operatorname{atan}\left(\tau_{\mathbf{a}} / \sigma_{\mathbf{a}}\right)(\mathbf{r a d s})$ & $\mathbf{s s f}$ \\
\hline 169 & 0 & 0.82 \\
142 & 0 & 0.70 \\
126 & 0 & 0.63 \\
120 & 0 & 0.60 \\
106 & 0 & 0.54 \\
101 & 0 & 0.52 \\
142 & 0.32 & 0.64 \\
124 & 0.32 & 0.47 \\
113 & 0.32 & 0.37 \\
109 & 0.32 & 0.33 \\
99 & 0.32 & 0.25 \\
95 & 0.32 & 0.21 \\
144 & 0.52 & 0.39 \\
110 & 0.52 & 0.33 \\
91 & 0.52 & 0.30 \\
84 & 0.52 & 0.28 \\
69 & 0.52 & 024 \\
64 & 0.52 & 0.23 \\
85 & 0.79 & 0.62 \\
68 & 0.79 & 0.46 \\
59 & 0.79 & 0.36 \\
55 & 0.79 & 0.31 \\
47 & 0.79 & 0.22 \\
44 & 0.79 & 0.18 \\
0 & 1.57 & 0.8 \\
\hline
\end{tabular}

The first column shows the normal stress amplitude followed by the stress amplitude ratio given by $\lambda=\operatorname{atan}\left(\tau_{\mathrm{a}} / \sigma_{\mathrm{a}}\right)$ and respective ssf. The stress amplitude ratio aims to differentiate proportional stress paths based on their normal and shear stress amplitudes.

This ratio is the tangent of the angle between normal and shear stress amplitudes de-picted in the von Mises stress space, and the atan of this ratio gives the angle itself, which improves the identification of the stress path. For the loading cases considered in this study, the ratio of stress amplitudes varies from 0 to 1.57 , i.e., case 1 has $\lambda=0$; case $2 \lambda=1.57$; case $3 \lambda=0.32$; case $4 \lambda=0.52$; and finally, case $5 \lambda=0.79$.

The regression study was conducted using Datafit software, version 9.1 (Oakdale Engineering, Oakdale, Pennsylvania, USA). This software has a large database of multivariable equations that are evaluated for a given data set, e.g., Table 9. These equations are then prioritized according to their quality of fit, i.e., the equations are prioritized according to decreasing values of $R^{2}$; equations with higher $R^{2}$ fit the experimental results better and therefore come first in this prioritization. Next, the graph of the equation that best fits the results must be analyzed to verify that the equation matches the expected result, i.e., the mechanical behavior expected for materials subjected to cyclic loading. If it does not, the search continues until the correct equation is found.

These methods were applied to Table 9 and as a result Equation (2) was obtained to model the ssf variation of magnesium alloy AZ31B-F. The $R^{2}$ of this equation is 0.93 , which is a map that sets the damage scale between normal and shear stresses according to their stress amplitude ratio and normal stress amplitudes, which can be considered as a material property.

$$
\operatorname{ssf}_{A Z 31 B F}=a+b \cdot \sigma_{a}+c \cdot \sigma_{a}{ }^{2}+d \cdot \sigma_{a}{ }^{3}+e \cdot \sigma_{a}{ }^{4}+f \cdot \sigma_{a}{ }^{5}+g \cdot \lambda+h \cdot \lambda^{2}+i \cdot \lambda^{3}+j \cdot \lambda^{4}
$$

The input variables of Equation (2) are given in $\mathrm{MPa}$ and in radians, and the equation constants $a$ to $j$ are given in Table 10 . 
Table 10. Constant values of Equation (2) for the condition of best fit $\left(R^{2}=0.93\right)$-AZ31BF damage map.

\begin{tabular}{cc}
\hline Variable & Value \\
\hline$a$ & 3.00516795748732 \\
$b$ & 0.138210394574867 \\
$c$ & $2.11406573677796 \times 10^{-3}$ \\
$d$ & $1.51576767021405 \times 10^{-5}$ \\
$e$ & $-4.82672910096113 \times 10^{-8}$ \\
$f$ & $5.11194628585213 \times 10^{-11}$ \\
$g$ & -0.518185467569207 \\
$h$ & -1.19420385023642 \\
$i$ & 2.98394174345283 \\
$j$ & -0.655924758831285 \\
\hline
\end{tabular}

Figure 6a shows the AZ31B-F ssf variation for the four load paths considered. To improve the comparison between the loading paths, the normal stress amplitudes were divided by their respective maximum values. As can be seen for $42 \mathrm{CrMo} 4$ [21], the trend lines correlating ssf and normal stresses have different slopes for different stress amplitude ratios, which means that the damage scale between normal and shear stress amplitudes is not constant and varies depending on the loading path (different trend lines in Figure 6a) and depending on the normal stress amplitude (different slopes of the trend lines). Figure $6 \mathrm{~b}$ shows the graph of Equation (2) represented with a color gradient and the data listed in Table 9 represented by black dots. The trend lines shown in Figure 6a fit these black dots, i.e., they are the expected intersection of the graph shown in Figure $6 \mathrm{~b}$ for the stress amplitude ratios considered in the experiments.

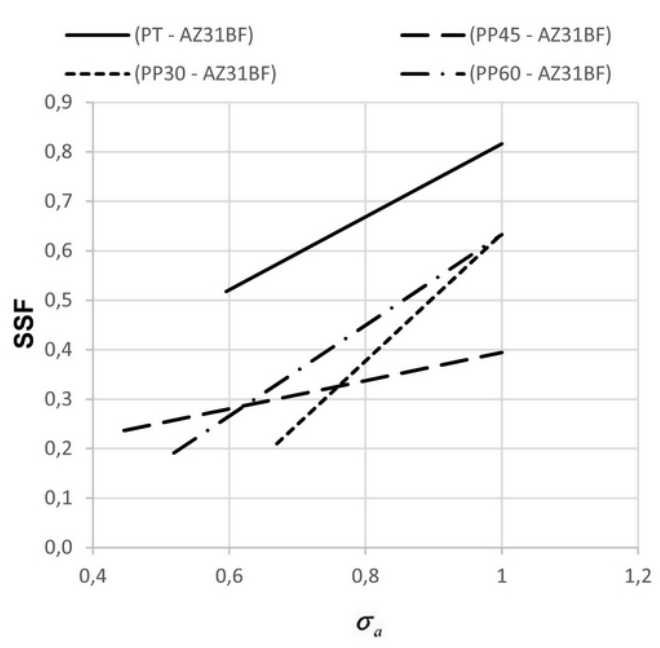

(a)

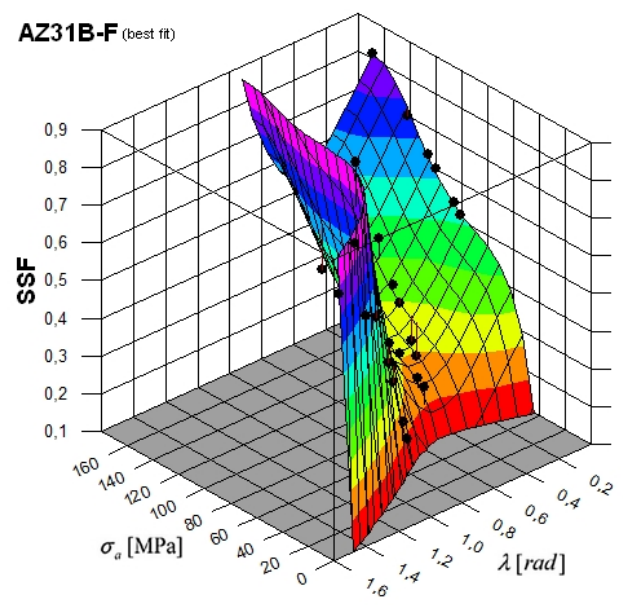

(b)

Figure 6. ssf variation for the AZ31B-F magnesium alloy. (a) ssf trend lines based on experimental data, (b) Tridimensional graph of Equation (2) representing the ssf variation according to the normal stress amplitude and stress amplitude ratios-ssf damage map.

The objective of the AZ31B-F damage map represented by Equation (2) is to determine the ssf for stress amplitude ratios other than those considered in the experiments, since it is almost impossible to obtain these data for all possible stress amplitude ratios through experiments. An important aspect to be analyzed when selecting and validating a ssf function is the ssf transitions between experimental stress amplitude ratios. The ssf aestimates for stress amplitude ratios that were not included in the experiments must be consistent with the experimental data. This means that it is not sufficient to use only the quality of fit parameters to select the ssf functions, but it is necessary to verify the function transitions 
and infer the quality of their ssf estimates. Figure $6 \mathrm{~b}$ shows the best fit for the experimental ssf data after analyzing the transitions between the estimates and the experiments. As we can see, the transitions are smooth and do not change direction abruptly; moreover, the ssf function is defined in all domains. These features together with a high $\mathrm{R}^{2}$ led us to choose Equation (2) as the damage map for the AZ31B-F. Despite the high $\mathrm{R}^{2}$ of Equation (2), other equations from the database considered in this study have better $\mathrm{R}^{2}$, but their ssf transitions do not represent the expected mechanical ssf behavior or they were not defined in all domains.

Equation (3) shows the ssf damage map for the high strength steel 42CrMo4 [21], which was used to demonstrate the ssf theory. As you can see, Equation (2) has a different expression with ten constants compared to the eight constants of Equation (3).

$$
s s f_{42 \mathrm{CrMo} 4}=a+b \cdot \sigma_{a}+c \cdot \sigma_{a}{ }^{2}+d \cdot \sigma_{a}{ }^{3}+f \cdot \lambda^{2}+g \cdot \lambda^{3}+h \cdot \lambda^{4}+i \cdot \lambda^{5}
$$

Equation (2) represents the best fit for the AZ31B-F material. However, the original ssf equation (Equation (3)) is still valid and can also be applied to AZ31B-F, but with a lower $\mathrm{R}^{2}$. Table 11 shows the constants of Equation (3) (42CrMo4 expression) obtained for the AZ31B-F material.

Table 11. Regression variable results for AZ31BF damage map $\left(R^{2}=0.9\right)$ for the condition of the $42 \mathrm{CrMo} 4$ fit equation type given by Equation (3).

\begin{tabular}{cc}
\hline Variable & Value \\
\hline$a$ & -0.759474996569004 \\
$b$ & $2.80999535970111 \times 10^{-2}$ \\
$c$ & $-2.13782035002517 \times 10^{-4}$ \\
$d$ & $6.13142129539061 \times 10^{-7}$ \\
$f$ & -5.34317526860744 \\
$g$ & 14.1883590988772 \\
$h$ & -11.7431172616598 \\
$i$ & 3.26772185827734 \\
$j$ & -0.759474996569004 \\
\hline
\end{tabular}

The $\mathrm{R}^{2}$ obtained for the AZ31B-F using the $42 \mathrm{CrMo} 4$ expression was 0.9 against 0.93 obtained with the best fit equation approach. The difference between these two expressions regarding the $\mathrm{R}^{2}$ can be considered negligible which means that the original ssf expression, Equation (3), can be used to model the ssf variation in materials very different from the 42CrMo4 material, such as the Az31B-F magnesium alloy. This idea is reinforced by comparing Figure $6 \mathrm{~b}$ with Figure $7 \mathrm{a}$ where it can be seen a strong similarity between the two graphs. Figure 7 shows the ssf graphs for both materials considering Equation (3), i.e., considering the $42 \mathrm{CrMo} 4 \mathrm{ssf}$ expression. Observing this figure one can conclude that there is a mirroring between these two graphs, however the surface shape for both materials is similar. This behavior results from a kind of SFF symmetric variation in both materials, to better describe this, Figure 8 shows these variations for both materials according to stress amplitude ratios. 


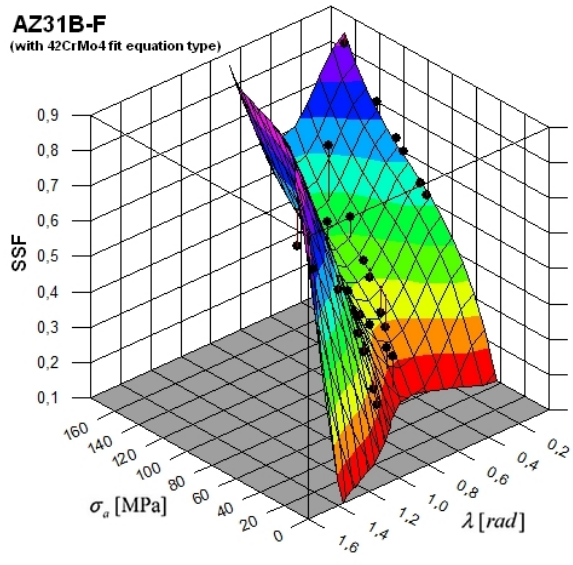

(a)

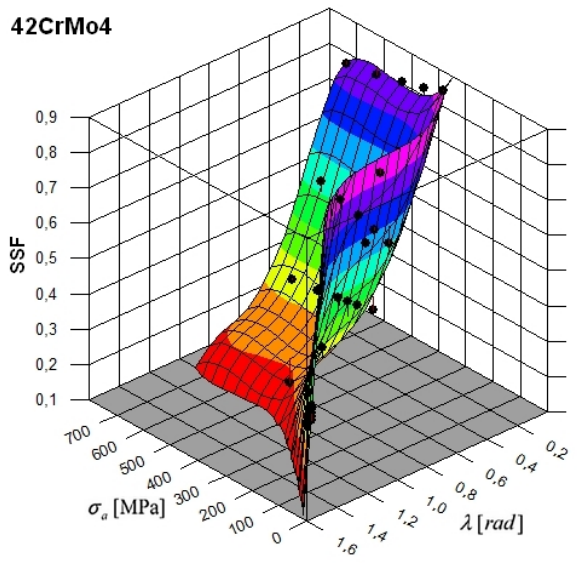

(b)

Figure 7. ssf graph of both materials using the $42 \mathrm{CrMo} 4$ fit equation (Equation (3). (a) Case of AZ31B-F magnesium alloy, (b) Case of 42CrMo4 high strength material.

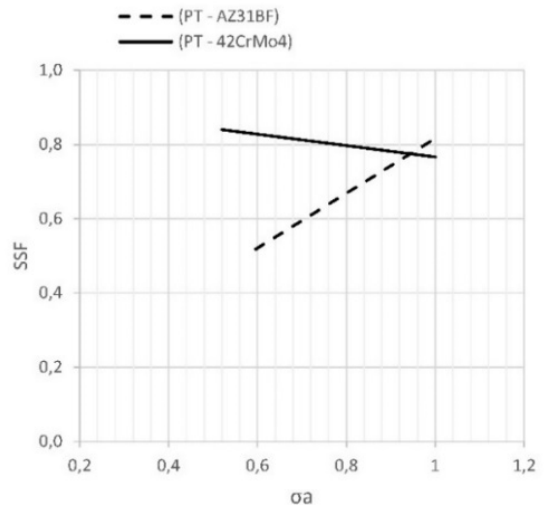

(a)

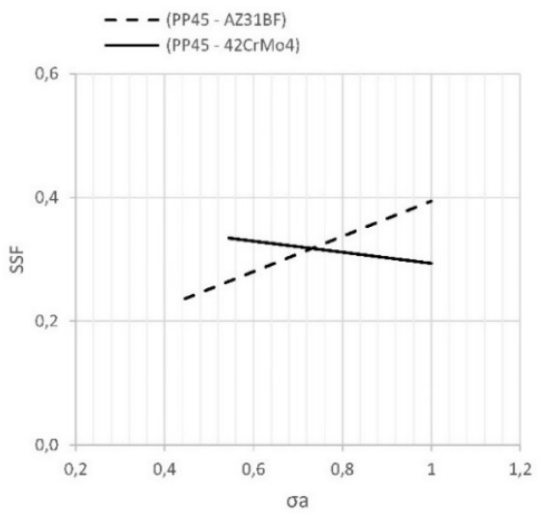

(c)

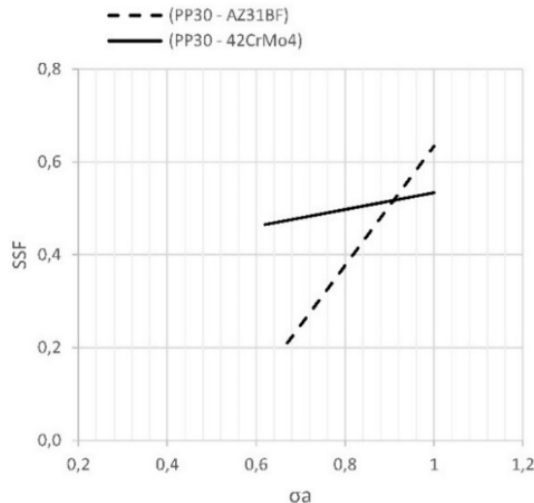

(b)

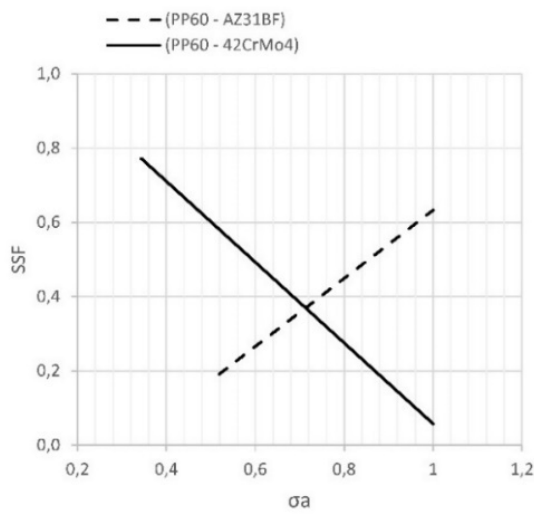

(d)

Figure 8. ssf experimental results for AZ31B-F and 42CrMo4. (a) Case 1-PT pure tension, (b) Case 3-PP30, (c) Case 4-PP45, and (d) Case 5-PP60.

Figure $8 \mathrm{a}-\mathrm{d}$ shows the variation of ssf as a function of variation of normal stress for both materials. From these results, it can be concluded that in cases 1, 4, and 5, the trend lines of both materials have slopes with different signs. For example, in Figure 8a, case 1-PT, the ssf increases when the normal stresses in AZ31B-F increase. On the other hand, the ssf decreases when the normal stresses in $42 \mathrm{CrMo} 4$ increase. This means that the contribution of normal stress amplitudes to the total damage (damage due to shear 
stress amplitudes plus damage due to normal stress amplitudes) is weighted differently depending on the material and fatigue state (LCF or HCF). In all subframes of Figure 8, the $42 \mathrm{CrMo} 4$ trend lines lie above the Az31B-F trend lines for dimensionless normal stresses near 0.6; this means that under the HCF regime, the normal stress amplitude has a higher contribution to the total damage in the 42CrMo4 material compared to AZ31B-F. On the other hand, in the LCF regime, the opposite is true, i.e., the amplitude of the normal stress has a higher contribution to the aggregate damage in AZ31B-F than in $42 \mathrm{CrMo} 4$. This behavior is the reason for the mirror image in the plots in Figure 7. The contribution of normal stresses to the aggregate damage in magnesium alloy AZ31B-F is larger in LCF than in HCF. Therefore, the role of shear stress amplitudes in fatigue damage increases as the amplitudes of normal and shear stresses decrease, i.e., in the threshold region between finite and infinite life, shear stress amplitude will be the dominant stress component. Figure 9 shows the aerial view of Figure 7, showing the correlation between the normal stresses and the stress amplitude ratios, with the colors indicating the ssf variation. In this figure, the lower grey area shows the infinite life diagram area and the upper area above this grey area bounds the finite fatigue life area. Based on this grey area, a model can be created that establishes a boundary between finite and infinite life for proportional loads.

AZ31B-F (best fit)

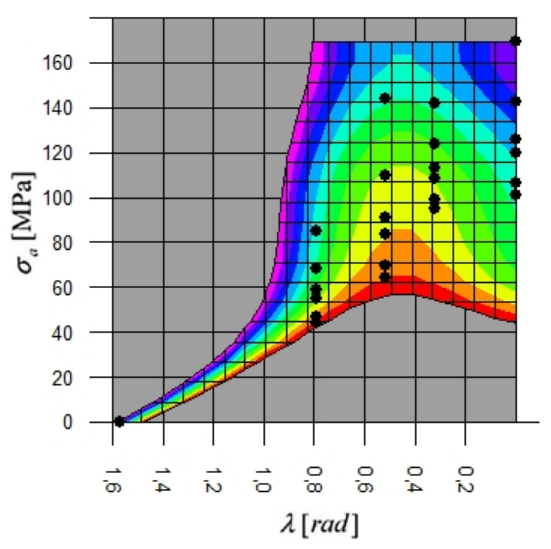

(a)

\section{$42 \mathrm{CrMo4}$}

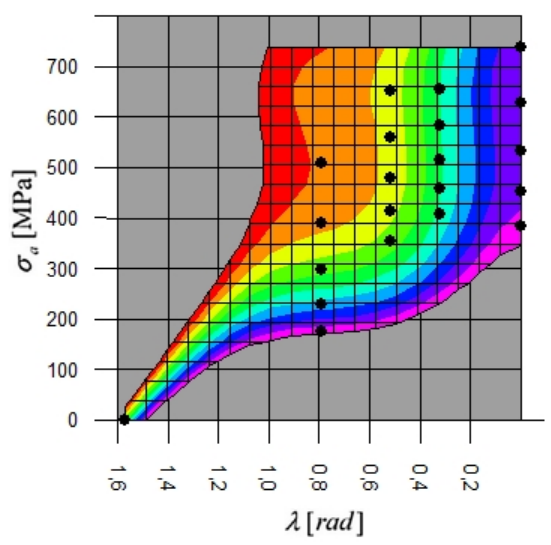

(b)

Figure 9. Normal stress vs. stress amplitude ratio, (a) AZ31B-F, (b) 42CrMo4.

Figure 10 shows the threshold model for the AZ31B-F material, where each point represents the normal stress amplitude at $10^{6}$ cycles (infinite life threshold) versus the respective stress amplitude ratio. The line shown in the graph is obtained by creating a linear trend line over the data of the graph. An offset is then made to place all points above the trend line. In this way, it becomes possible to obtain a simple boundary where a safe region can be identified by the trend line equation, as shown in Figure 10 and Equation (4).

The safe/unsafe region approach can be used in the design of AZ31B-F material structures or components subjected to proportional loads with any combination of shear and normal stress amplitudes. In this approach, Equation (4) is used to calculate the maximum value of $\sigma_{a}$ for a given $\lambda$. Then, Equation (5) can be used to determine the respective shear stress amplitude. 


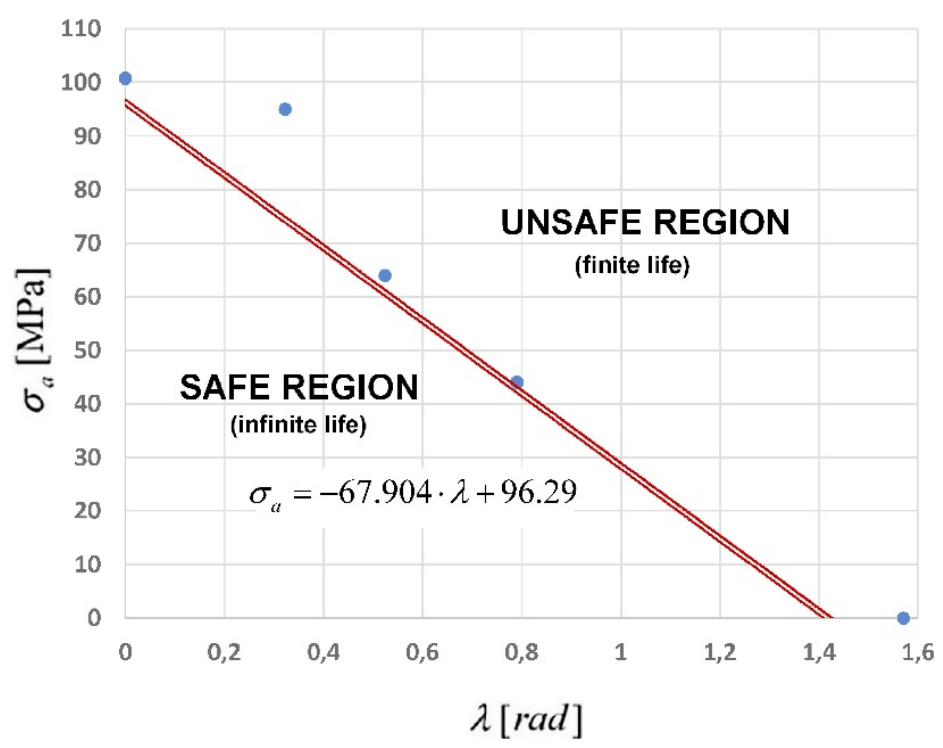

Figure 10. AZ31B-F safe region identification for proportional loading conditions.

$$
\begin{gathered}
\sigma_{a_{-} t h}=96.29-67.90 \cdot \lambda \\
\tau_{a_{-} t h}=\sigma_{a_{-} t h} \cdot \tan (\lambda)
\end{gathered}
$$

These two values, $\sigma_{a_{-} t h}$ and $\tau_{a_{-} t h}$, calculated in this way, represent the maximum amplitudes allowed for infinite life condition, where th denotes threshold. In this sense, it is possible to develop an expression to estimate the safety factor in terms of infinite life for a given proportional load. To do this, Equation (1) is used to obtain the threshold value of the ssf equivalent shear stress, and then the same equation is used to obtain the ssf equivalent shear stress for the loading $\left(\sigma_{a}, \tau_{a}\right)$ as seen in Equation (6) where $\tau_{a}$ is replaced by $\sigma_{a} \cdot \tan (\lambda)$.

$$
n=\frac{\sigma_{a_{-} t h} \cdot \tan (\lambda)+s s f \cdot \sigma_{a_{\_} t h}}{\sigma_{a} \cdot \tan (\lambda)+s s f \cdot \sigma_{a}}
$$

simplifying Equation (6) we get Equation (7)

$$
n=\frac{\sigma_{a-t h}}{\sigma_{a}}
$$

replacing $\sigma_{a_{-} t h}$ by its expression in Equation (7) it is obtained the safety factor expression for the AZ31B-F magnesium alloy subjected to proportional loadings, Equation (8), where $\sigma_{a}$ is the normal stress amplitude of a given proportional loading and $\lambda$ is its stress amplitude ratio.

$$
n=\frac{96.29-67.90 \cdot \lambda}{\sigma_{a}}
$$

\section{Conclusions}

In this work, the cyclic behavior of the AZ31B-F was analyzed using the methodology developed by Anes et al. for the high strength steel 42CrMo4. The stress paths described in this methodology were implemented in the laboratory for the AZ31B-F and the results were subsequently analyzed. To extrapolate the experimental results to a wide range of stress amplitude ratios, a regression was performed over the experimental data resulting in the AZ31B-F damage map (ssf). The main conclusions were as follows:

1. The ssf damage map has been successfully evaluated for AZ32BF and can be used to estimate the fatigue life of AZ31BF components and structures under proportional loads. 
2. As for $42 \mathrm{CrMo} 4$, the damage scale between normal and shear stresses (ssf) in AZ31B-F material varies depending on the stress amplitude ratio and stress intensity, but the pattern of this variation is different from $42 \mathrm{CrMo} 4$ material.

3. The regression study showed that the $42 \mathrm{CrMo} 4$ expression for the damage map was also valid for AZ31B-F material, but with a lower $\mathrm{R}^{2}$ compared to the $\mathrm{R}^{2}$ obtained for the best fit condition.

4. It is concluded that the polynomial function with eight constants initially obtained for the $42 \mathrm{CrMo} 4$ material is a good candidate for modelling the ssf damage map of a variety of materials, but further studies are needed to confirm this hypothesis.

5. Based on the AZ31B-F damage map, an expression was developed to calculate the safety factor of AZ31B-F under proportional loading. This safety factor was developed with respect to infinite life conditions.

Future work is planned to include non-proportional effects in the damage map and safety factor for infinite life. To the best of the author's knowledge, the non-proportional loading effects for various stress amplitude ratios are not yet known for the AZ31B-F material.

Author Contributions: Conceptualization, V.A. and L.R.; methodology, V.A. and L.R.; software, V.A.; validation, L.R. and M.F.; formal analysis, L.R.; investigation, V.A.; resources, L.R.; data curation, V.A.; writing—original draft preparation, V.A.; writing—review and editing, L.R.; visualization, V.A.; supervision, L.R.; project administration, L.R.; funding acquisition, M.F. All authors have read and agreed to the published version of the manuscript.

Funding: This work was supported by FCT, through IDMEC, under LAETA, project UIDB/50022/2020, and also by Polytechnic Institute of Lisbon through the Projects for Research, Development, Innovation and Artistic Creation (IDI\&CA), within the framework of the project ReEdIA—Risk Assessment and Management in Open Innovation, IPL/2021/ ReEdIA/ISEL.

Institutional Review Board Statement: Not applicable.

Informed Consent Statement: Not applicable.

Data Availability Statement: Data sharing is not applicable to this article.

Conflicts of Interest: The authors declare no conflict of interest.

\section{References}

1. Tolliver, C.; Keeley, A.R.; Managi, S. Drivers of Green Bond Market Growth: The Importance of Nationally Determined Contributions to the Paris Agreement and Implications for Sustainability. J. Clean. Prod. 2020, 244, 118643. [CrossRef]

2. Salawitch, R.J.; Canty, T.P.; Hope, A.P.; Tribett, W.R.; Bennett, B.F. Paris Climate Agreement: Beacon of Hope; Springer Nature: London, UK, 2017; pp. 1-35.

3. Aghion, E.; Bronfin, B.; Eliezer, D. The Role of the Magnesium Industry in Protecting the Environment. J. Mater. Process. Technol. 2001, 117, 381-385. [CrossRef]

4. Kulekci, M.K. Magnesium and Its Alloys Applications in Automotive Industry. Int. J. Adv. Manuf. Technol. 2008, 39, 851-865. [CrossRef]

5. Dai, Y.; Chen, X.-H.; Yan, T.; Tang, A.-T.; Zhao, D.; Luo, Z.; Liu, C.-Q.; Cheng, R.-J.; Pan, F.-S. Improved Corrosion Resistance in AZ61 Magnesium Alloys Induced by Impurity Reduction. Acta Metall. Sin. 2020, 33, 225-232. [CrossRef]

6. Yao, H.-L.; Yi, Z.-H.; Yao, C.; Zhang, M.-X.; Wang, H.-T.; Li, S.-B.; Bai, X.-B.; Chen, Q.-Y.; Ji, G.-C. Improved Corrosion Resistance of AZ91D Magnesium Alloy Coated by Novel Cold-Sprayed Zn-HA/Zn Double-Layer Coatings. Ceram. Int. 2020, 46, 7687-7693. [CrossRef]

7. Zeng, R.; Zhang, J.; Huang, W.; Dietzel, W.; Kainer, K.U.; Blawert, C.; Wei, K.E. Review of Studies on Corrosion of Magnesium Alloys. Trans. Nonferr. Met. Soc. China 2006, 16, s763-s771. [CrossRef]

8. Albinmousa, J.; Jahed, H. Multiaxial Effects on LCF Behaviour and Fatigue Failure of AZ31B Magnesium Extrusion. Int. J. Fatigue 2014, 67, 103-116. [CrossRef]

9. Albinmousa, J.; Jahed, H.; Lambert, S. Cyclic Axial and Cyclic Torsional Behaviour of Extruded AZ31B Magnesium Alloy. Int. J. Fatigue 2011, 33, 1403-1416. [CrossRef]

10. Albinmousa, J.; Jahed, H.; Lambert, S. Cyclic Behaviour of Wrought Magnesium Alloy under Multiaxial Load. Int. J. Fatigue 2011, 33, 1127-1139. [CrossRef]

11. Xiong, Y.; Yu, Q.; Jiang, Y. Multiaxial Fatigue of Extruded AZ31B Magnesium Alloy. Mater. Sci. Eng. A 2012, 546, 119-128. [CrossRef] 
12. Yu, Q.; Zhang, J.; Jiang, Y.; Li, Q. Multiaxial Fatigue of Extruded AZ61A Magnesium Alloy. Int. J. Fatigue 2011, $33,437-447$. [CrossRef]

13. Wang, Y.; Culbertson, D.; Jiang, Y. An Experimental Study of Anisotropic Fatigue Behavior of Rolled AZ31B Magnesium Alloy. Mater. Des. 2020, 186, 108266. [CrossRef]

14. Wang, F.; Feng, M.; Jiang, Y.; Dong, J.; Zhang, Z. Cyclic Shear Deformation and Fatigue of Extruded Mg-Gd-Y Magnesium Alloy. J. Mater. Sci. Technol. 2020, 39, 74-81. [CrossRef]

15. Zhao, X.; Gao, P.; Zhang, Z.; Wang, Q.; Yan, F. Fatigue Characteristics of the Extruded AZ80 Automotive Wheel. Int. J. Fatigue 2020, 132, 105393. [CrossRef]

16. Liu, Y.; Chen, Y.; He, C.; Liu, F.; Yang, K.; Li, L.; Zhang, H.; Wang, C.; Wang, Q. Vacuum Retarding and Air Accelerating Effect on the High-Cycle and Very-High-Cycle Fatigue Behavior of a ZK60 Magnesium Alloy. Mater. Des. 2020, 109310. [CrossRef]

17. Anes, V.; Reis, L.; Li, B.; Freitas, M. Crack Path Evaluation on HC and BCC Microstructures under Multiaxial Cyclic Loading. Int. J. Fatigue 2014, 58, 102-113. [CrossRef]

18. Anes, V.; Reis, L.; de Freitas, M. Evaluation of a Phenomenological Elastic-Plastic Approach for Magnesium Alloys under Multiaxial Loading Conditions. Fatigue Fract. Eng. Mater. Struct. 2019, 42, 2468-2486. [CrossRef]

19. Anes, V.; Reis, L.; Freitas, M. Evaluation of the AZ31 Cyclic Elastic-Plastic Behaviour under Multiaxial Loading Conditions. Frat. Ed. Integrità Strutt. 2014, 8, 282-292. [CrossRef]

20. Jahed, H.; Albinmousa, J. Multiaxial Behaviour of Wrought Magnesium Alloys-A Review and Suitability of Energy-Based Fatigue Life Model. Theor. Appl. Fract. Mech. 2014, 73, 97-108. [CrossRef]

21. Anes, V.; Reis, L.; Li, B.; Fonte, M.; De Freitas, M. New Approach for Analysis of Complex Multiaxial Loading Paths. Int. J. Fatigue 2014, 62, 21-33. [CrossRef]

22. Socie, D.F.; Marquis, G.B. Multiaxial Fatigue; Society of Automotive Engineers: Warrendale, PA, USA, 2000.

23. Anes, V.; De Freitas, M.; Reis, L. The Damage Scale Concept and the Critical Plane Approach. Fatigue Fract. Eng. Mater. Struct. 2017, 40, 1240-1250. 